\title{
La dose fait le poison*
}

\author{
par Marc M. Perreault
}

$\mathrm{L}$ e traitement liquidien par voie intraveineuse est omniprésent dans les hôpitaux. Il fait partie des ordonnances d'admission de tout patient, que ce soit sous la forme d'un traitement de réanimation, de soins d'urgence ou de soins postopératoires ou pour les patients atteints de sepsis ou encore comme traitement d'entretien destiné aux patients qui sont dans l'impossibilité de prendre des médicaments oraux; mais les solutions intraveineuses peuvent aussi servir à diluer la plupart des médicaments à usage parentéral. Normalement, le service de pharmacie n'achète ni ne stocke les liquides intraveineux et nous, pharmaciens d'hôpitaux, ne les considérons habituellement pas comme des médicaments, c'est-à-dire, comme un produit pourvu d'une relation dose-effet ou comme une cause potentielle de danger pour nos patients. Malgré la présence des lignes directrices de l'UK National Institute for Health and Care Excellence (publiées en 2013 et mises à jour en 2017) ${ }^{1}$, qui décrivent les principes généraux de gestion des liquides administrés par voie intraveineuse, ceux-ci sont, dans l'ensemble, toujours mal prescrits ${ }^{2,3}$. Il est temps pour nous, pharmaciens, de changer notre perspective en ce qui concerne les liquides et de commencer à considérer cette forme de traitement comme n'importe quelle autre pharmacothérapie, c'est-à-dire, un traitement nécessitant une individualisation et un suivi adéquat.

Un modèle conceptuel pour le traitement liquidien a été proposé afin d'en éviter l'utilisation inadéquate ${ }^{4}$. Ce modèle compte quatre phases distinctes de traitement liquidien : la première étant le secours (réanimation), suivie de l'optimisation et de la stabilisation et enfin la désescalade, un processus qui s'ajuste avec le temps à la diminution de la gravité de la maladié. Au cours de chacune des phases, l'individualisation (c'est-à-dire le type de liquide et la quantité administrée) est nécessaire au maintien de l'irrigation de l'organe tout en réduisant de façon significative le " troisième secteur ». Or, on est de plus en plus conscient des conséquences néfastes de donner une trop grande quantité de liquide, voire même d'administrer aux patients une trop grande quantité d'un même liquide (par exemple, la solution physiologique salée [chlorure de sodium à $0,9 \%$ ] $)^{5-10}$.

Nous avons tous vu ou vécu des cas où un excès de liquide avait été administré, par exemple à des patients admis au service des urgences qui se retrouvent quelques jours plus tard dans le service d'une autre unité médicale ou à l'unité de soins chirur-

*Paracelsus, dritte defensio [Troisième défense], 1538.

gicaux et qui présentent un excédent de liquide de cinq, voire même de dix litres. Ces patients ont habituellement subi des traitements de réanimation énergiques à l'aide de bolus liquidiens et ils reçoivent aussi des liquides comme traitement d'entretien; alors que leur état hémodynamique se stabilise et que la cascade inflammatoire régresse, la diurèse se déclenche d'elle-même ou une intervention est nécessaire pour la déclencher (à l'aide, par exemple, de l'administration d'un diurétique de l'anse ou de l'amorce d'un traitement de suppléance rénale). Malheureusement, cette approche semble être celle utilisée par défaut et produire le résultat auquel on s'attend pendant un séjour hospitalier, autrement dit : "Le patient doit enfler avant d'aller mieux. »

Cette utilisation énergique des liquides a été encouragée par le recours à des traitements précoces orientés vers des objectifs visant à fournir des liquides et des vasopresseurs selon les protocoles prédéfinis pour la gestion des sepsis sévères et chocs septiques ${ }^{11}$. La mise en œuvre rapide de tels protocoles a mené à des améliorations significatives des résultats cliniques ${ }^{11}$ et est actuellement l'une des meilleures pratiques dans la campagne "Surviving Sepsis (survivre au sepsis) $^{12}$.

Or, on constate l'apparition de nouvelles données sur les dommages potentiels associés au fait d'administrer trop de liquides (bilan hydrique positif) à des patients gravement malades. Plusieurs études rétrospectives ont découvert une association entre les cas de bilan hydrique cumulatif positif au moment du congé de l'unité de soins intensifs (USI) et les cas de décès (à l'USI ou ailleurs dans l'hôpital) ${ }^{5,6}$, ce qui laisse entrevoir que le fait d'intervenir sur le bilan hydrique pourrait améliorer les résultats thérapeutiques ${ }^{7}$. Jusqu' ici, il ne s'agit que d'une association et non d'un lien de causalité; cependant, de plus en plus de données probantes vont dans ce sens. Lors d'une analyse systématique et d'une méta-analyse d'études contrôlées à répartition aléatoire et d'études observationnelles, Silversides et collab. ${ }^{8}$ ont montré qu'une stratégie plus prudente concernant les liquides pour les patients atteints de sepsis ou d'un syndrome de détresse respiratoire aiguë permet d'accroître le nombre de journées sans respirateur et de réduire la durée des séjours à l'USI sans qu'il y ait de changement aux taux de mortalité, comparativement à une approche plus libre concernant les liquides ou des soins classiques, ce qui jette les bases d'importantes études à répartition aléatoire dans le but de déterminer la stratégie optimale concernant les liquides pour les maladies graves. 
Dans la plupart des cas, le liquide de choix pour la réanimation, l'entretien et la dilution de médicaments demeurait la solution physiologique salée, aussi appelée "liquide physiologique ». Il contient $154 \mathrm{mmol}$ de chlorure et de sodium et possède un $\mathrm{pH}$ de 5,5. Il n'est donc en rien physiologique et il devrait donc être considéré comme anormal et non "normal " comme le veut le terme anglais "normal saline ». Un trouble métabolique bien connu, qui est produit par l'administration d'une trop grande quantité de solution physiologique salée, est l'hyperchlorémie et l'acidose métabolique à trou non anionique qui lui est associée. Au cours de la dernière décennie, on a reconnu qu'une surdose de chlorure provenant de solution physiologique salée a le potentiel de provoquer l'insuffisance rénale aiguë. Cependant, deux importantes études cliniques comparant la solution physiologique salée aux solutions cristalloïdes équilibrées ne sont pas arrivées à prouver l'existence d'un tel lien ${ }^{9,10}$. Pour l'instant, il reste à déterminer quel est le cristalloïde optimal.

Je ne prétends évidemment pas résoudre les controverses associées au traitement liquidien, mais je cherche à mettre de l'avant l'accumulation de données indiquant qu'un excès de liquides (en général) et trop de solution physiologique salée (en particulier) ne représentent pas une pharmacothérapie optimale. Cependant, les doses optimales de liquides et de solution physiologique salée pour un patient restent inconnues.

Par ailleurs, il y a des enjeux précis concernant les liquides auxquels nous, pharmaciens, faisons face et qui méritent d'être abordés. Le premier est la nécessité de reconnaitre à quel moment on doit alléger un traitement liquidien, tout comme il est nécessaire d'accélérer le passage à un antibiotique à spectre plus étroit pour un patient dont l'infection se résorbe progressivement. Il y a des indicateurs spécifiant le moment de l'administration d'un bolus de liquide, dont un choc, une chute de pression artérielle systolique ou une augmentation du taux de lactate sérique. Par contre, il n'y a toujours pas d'indicateurs définis signalant le moment de réduire ou de cesser l'administration de liquide d'entretien. Selon moi, l'amorce de l'administration de diurétiques par l'équipe sert de repère indiquant qu'il est temps de réévaluer le traitement d'entretien. Malheureusement, sans de tels indicateurs, l'infusion de liquides se poursuit plus longtemps que nécessaire et les patients subissent une surdose de fluides encore plus importante.

Un deuxième enjeu apparaissant dans la littérature est la place des liquides utilisés pour diluer les médicaments dans le bilan hydrique total. Dans une importante étude rétrospective menée auprès de patients gravement malades au Royaume-Uni et au Canada, les raisons motivant l'administration de liquides pendant les jours 1 à 3 aux USI étaient, étonnamment, en premier lieu la dilution de médicaments $(34,5 \%$ de tous les liquides) alors que le traitement d'entretien et les bolus comptaient respectivement pour $26,5 \%$ et $24,4 \%$ des liquides ${ }^{7}$. Une observation semblable a été faite auprès d'une population à l'USI médicale, où les diluants de médicaments comptaient pour $63 \%$ du volume parentéral total dans les sept premiers jours suivant l'admission à l'USI et étaient responsables d'une plus grande incidence d'hyperchlorémie ${ }^{13}$. Ainsi, pour limiter l'administration de liquides, les pharmaciens doivent tenir compte du poids des diluants dans le bilan hydrique total et aussi participer à l'élaboration de stratégies de restriction de l'utilisation des liquides.
Par conséquent, la prochaine fois que vous participerez à une tournée médicale, prenez le temps de réévaluer le traitement d'entretien et songez à administrer les médicaments à l'aide de plus faibles volumes de diluants, si possible, ou à passer de médicaments administrés par voie intraveineuse à des médicaments administrés par voie orale ou parentérale. Ces précautions empêcheront les liquides de devenir des poisons!

[Traduction par l'éditeur]

Références

1. Intravenous fluid therapy for adults in hospital [clinical guideline CG174]. Londres (Royaume-Uni) : National Institute for Health and Care Excellence (NICE); 2013 [mis à jour en mai 2017]. Publié au : https://www.nice.org.uk/ guidance/cg174. Consulté le 4 janvier 2019.

2. Gao X, Huang KP, Wu HY, Sun PP, Yan JJ, Chen J, et al. Inappropriate prescribing of intravenous fluid in adult inpatients-a literature review of current practice and research. J Clin Pharm Ther. 2015;40(5):489-95.

3. Sansom LT, Duggleby L. Intravenous fluid prescribing: improving prescribing practices and documentation in line with NICE CG174 guidance. BMJ Open Qual. 2014;3(1):u205899.w2409.

4. Hoste EA, Maitland K, Brudney CS, Mehta R, Vincent JL, Yates D, et al; ADQI XII Investigators Group. Four phases of intravenous fluid therapy: a conceptual model. Br J Anaesth. 2014;113(5):740-7.

5. Lee J, de Louw E, Niemi M, Nelson R, Mark RG, Celi LA, et al. Association between fluid balance and survival in critically ill patients. J Intern Med. 2015;277(4):468-77.

6. Brotfain E, Koyfman L, Toledano R, Borer A, Fucs L, Galante O, et al Positive fluid balance as a major predictor of clinical outcome of patients with sepsis/septic shock after ICU discharge. Am J Emerg Med. 2016;34(11):2122-6.

7. Silversides JA, Fitzgerald E, Manickavasagam US, Lapinsky SE, Nisenbaum R, Hemmings N, et al.; Role of Active Deresuscitation After Resuscitation (RADAR) Investigators. Deresuscitation of patients with iatrogenic fluid overload is associated with reduced mortality in critical illness. Crit Care Med. 2018;46(10):1600-7.

8. Silversides JA, Major E, Ferguson AJ, Mann EE, McAuley DF, Marshall JC, et al. Conservative fluid management or deresuscitation for patients with sepsis or acute respiratory distress syndrome following the resuscitation phase of critical illness: a systematic review and meta-analysis. Intensive Care Med. 2017;43(2):155-70.

9. Young P, Bailey M, Beasley R, Henderson S, Mackle D, McArthur C, et al.; SPLIT Investigators; ANZICS CTG. Effect of a buffered crystalloid solution vs saline on acute kidney injury among patients in the intensive care unit: the SPLIT randomized clinical trial. JAMA. 2015;314(16):1701-10.

10. Semler MW, Self WH, Wanderer JP, Ehrenfeld JM, Wang L, Byrne DW, et al.; SMART Investigators. Balanced crystalloids versus saline in critically ill adults. N Engl J Med. 2018;378(9):829-39.

11. Rivers E, Nguyen B, Havstad S, Ressler J, Muzzin A, Knoblich B, et al. Early goal-directed therapy in the treatment of severe sepsis and septic shock. NEngl J Med. 2001;345(19):1368-77.

12. Rhodes A, Evans LE, Alhazzani W, Levy MM, Antonelli M, Ferrer R, et al. Surviving Sepsis Campaign: international guidelines for management of sepsis and septic shock 2016. Crit Care Med. 2017;45(3):486-552.

13. Magee C, Thompson-Bastin M, Laine M, Bissell B, Moran P, Owen G, et al. Insidious harm of medication diluents as a contributor to cumulative volume and hyperchloremia [résumé 12]. Crit Care Med. 2018;46(1 Suppl):6.

Marc M. Perreault, B. Pharm., M. Sc., Pharm. D., FCSHP, FOPQ, est pharmacien en soins critiques à l'Hôpital général de Montréal, Montréal, Québec. II est également rédacteur adjoint au Journal canadien de la pharmacie hospitalière.

Intérêts concurrents : Aucun déclaré.

Adresse de correspondance :

Dr Marc M.Perreault

Service de pharmacie

Hôpital général de Montréal

1650, avenue Cedar, bureau C1-200

Montréal (Québec) H3G 1A4

Courriel : marc.perreault@umontreal.ca 